\title{
First experience with the jump-starting robotic assistance device Cirq
}

\author{
Sandro M. Krieg, MD, MBA, and Bernhard Meyer, MD
}

Department of Neurosurgery, Klinikum rechts der Isar, Technische Universität München, Munich, Germany

Since spinal navigation became applicable, including robotic assistance into standard navigational setups seems reasonable. A newly released modular robotic assistance for drill stabilization (Cirq, Brainlab) was used in a 74-year-old man undergoing dynamic stabilization of L3-4 via navigated transfascial pedicle screws. The authors demonstrate the second worldwide surgery with this device and the second case performed in their department. They provide insight in its applicability to estimate its further potential in spinal robotics. Although being just the first step of this universal platform, the authors already see clinical benefit by its ease of use and drill support.

The video can be found here: https://youtu.be/oN2ZiHFRFkU.

KEYWORDS robotic assistance device; dynamic stabilization; guidance; lumbar spine; neuronavigation; video 